\title{
BMJ Rationing of total knee replacement: OPen a cost-effectiveness analysis on a large trial data set
}

\author{
Helen Dakin, ${ }^{1}$ Alastair Gray, ${ }^{1}$ Ray Fitzpatrick, ${ }^{2}$ Graeme MacLennan, ${ }^{3}$ \\ David Murray, ${ }^{4}$ The KAT Trial Group
}

To cite: Dakin H, Gray A, Fitzpatrick $\mathrm{R}$, et al. Rationing of total knee replacement: a cost-effectiveness analysis on a large trial data set. BMJ Open 2012;2: 000332. doi:10.1136/

bmjopen-2011-000332

- Prepublication history for this paper is available online. To view these files please visit the journal online (http:// bmjopen.bmj.com).

Protocol: Available at http://www.hta.ac. uk/protocols/199500100001. pdf

Received 26 August 2011 Accepted 21 December 2011

This final article is available for use under the terms of the Creative Commons Attribution Non-Commercial 2.0 Licence; see http://bmjopen.bmj.com

${ }^{1}$ Health Economics Research Centre, Department of Public Health, University of Oxford, Oxford, UK

${ }^{2}$ Department of Public Health, University of Oxford, Oxford, UK

${ }^{3}$ Health Services Research Unit, University of Aberdeen, Aberdeen, UK

${ }^{4}$ Nuffield Department of Orthopaedics, Rheumatology and Musculoskeletal Sciences, University of Oxford, Oxford, UK

Correspondence to Helen Dakin;

helen.dakin@dph.ox.ac.uk

\section{ABSTRACT}

Objectives: Many UK primary care trusts have recently introduced eligibility criteria restricting total knee replacement (TKR) to patients with low pre-operative Oxford Knee Scores (OKS) to cut expenditure. We evaluate these criteria by assessing the cost-effectiveness of TKR compared with no knee replacement for patients with different baseline characteristics from an NHS perspective.

Design: The cost-effectiveness of TKR in different patient subgroups was assessed using regression analyses of patient-level data from the Knee Arthroplasty Trial, a large, pragmatic randomised trial comparing knee prostheses.

Setting: 34 UK hospitals.

Participants: 2131 osteoarthritis patients undergoing TKR.

Interventions and outcome measures: Costs and quality-adjusted life years (QALYs) observed in the Knee Arthroplasty Trial within 5 years of TKR were compared with conservative assumptions about the costs and outcomes that would have been accrued had TKR not been performed.

Results: On average, primary TKR and 5 years of subsequent care cost $£ 7458$ per patient (SD: $£ 4058$ ), and patients gained an average of 1.33 (SD: 1.43) QALYs. As a result, TKR cost $£ 5623 /$ QALY gained. Although costs and health outcomes varied with age and sex, TKR cost $<£ 20000 /$ QLY gained for patients with American Society of Anaesthesiologists grades 1-2 who had baseline OKS $<40$ and for American Society of Anaesthesiologists grade 3 patients with OKS $<35$, even with highly conservative assumptions about costs and outcomes without TKR. Body mass index had no significant effect on costs or outcomes. Restricting TKR to patients with pre-operative OKS $<27$ would inappropriately deny a highly cost-effective treatment to $>10000$ patients annually.

Conclusions: TKR is highly cost-effective for most current patients if the NHS is willing to pay $£ 20000-£ 30000 /$ QALY gained. At least $97 \%$ of TKR patients in England have more severe symptoms than the thresholds we have identified, suggesting that further rationing by OKS is probably unjustified. Trial registration number: ISRCTN 45837371.

\section{ARTICLE SUMMARY}

\section{Article focus}

- We assess the cost-effectiveness of total knee replacement (TKR) compared with no knee replacement for patients with different baseline characteristics from a NHS perspective.

- In particular, we assess the appropriateness of eligibility criteria recently introduced by many UK primary care trusts, which restrict TKR to patients with low (ie, poor) pre-operative Oxford Knee Scores (OKS) to cut expenditure.

Key messages

- We find TKR to be highly cost-effective, costing $£ 5623$ per quality-adjusted life year gained for the average patient.

- TKR costs $<£ 20000$ per quality-adjusted life year gained for healthy patients with OKS of $<40$ or $<35$ for patients who have other conditions restricting their daily activities.

- We find no evidence to support the criteria for restricting access to TKR that have been proposed by some primary care trusts and calculate that restricting TKR to those patients with pre-operative OKS of 26 or less would deny a highly cost-effective treatment to $>10000$ patients/year.

Strengths and limitations of this study

- This is the first study assessing how the costeffectiveness of TKR varies with OKS and the first assessing the clinical/economic implications of the newly introduced rationing criteria.

- Analyses are based on patient-level data from a large pragmatic trial with detailed prospective collection of utilities, baseline characteristics and all major knee-related NHS resource use, including revisions and ambulatory care.

- Our study makes several highly conservative assumptions: in particular, assuming that patients would have accrued no knee-related costs and remained at baseline utility without TKR. Furthermore, the Knee Arthroplasty Trial sample included only 37 patients with preoperative OKS $>35$. As result, TKR may be also cost-effective for some patients with OKS above 39 . 


\section{INTRODUCTION}

Total knee replacement (TKR) is a highly effective treatment for patients with bone-on-bone osteoarthritis and significant knee symptoms, producing substantial reductions in symptoms (particularly pain) ${ }^{1}$ and lasting for at least 15 years in $83 \%-94 \%$ of cases. ${ }^{2}$ However, about $18 \%$ of patients consider the outcomes of their surgery to be only fair or poor, ${ }^{1}$ and a small proportion experience complications. ${ }^{3-5}$ Previous economic evaluations have found TKR to be highly cost-effective, with a cost-effectiveness ratio between $€ 1276$ and $\$ 18300$ per quality-adjusted life year (QALY) gained for the average patient $^{6-10}$ : well below the $£ 20000-£ 30000 /$ QALY range that the National Institute for Health and Clinical Excellence (NICE) considers cost-effective. ${ }^{11}$ However, studies suggest that the costs and benefits of TKR vary between patient subgroups, with TKR being more costeffective in younger patients, ${ }^{9}$ those attending highvolume centres ${ }^{7}$ and those deemed low-risk based on age, comorbidities and poverty criteria. ${ }^{7}$ There is also evidence that older ${ }^{12}$ and obese ${ }^{13}$ patients have higher medical costs, although other studies suggest that knee replacement is also cost-effective in non-agenarians ${ }^{14}$ and draw conflicting conclusions on how benefits vary with body mass index (BMI). ${ }^{15-17}$

In the current economic environment, there is great pressure to reduce NHS expenditure, and numerous Primary Care Trusts (PCTs) have proposed cutting costs by limiting access to joint replacement ${ }^{18-25}$ or classifying these procedures as being of limited value. ${ }^{24-26}$ Since around 60000 primary TKR procedures are conducted in England and Wales each year, ${ }^{27}$ savings could be made with little loss of patient welfare if it were possible to reliably predict which patients obtain little/no benefit from TKR based on explicit evidence-based criteria. Although the widely used Oxford Knee Score (OKS) was developed to assess outcomes of knee replacement in trial populations, ${ }^{28}$ several PCTs have set maximum OKS thresholds (ranging from 18 to 32), above which patients are ineligible for TKR. ${ }^{18-24}$ However, we are not aware of any clinical or cost-effectiveness justification for these thresholds. Furthermore, many patients with higher OKS have significant symptoms or limitations to daily activities that could be improved by surgery. If rationing policies are to be adopted, cost-effectiveness analysis offers the only fair basis for designing them since it ensures that NHS resources are allocated to maximise the health gained from available resources. As treatments are generally considered cost-effective if they cost $<£ 20000-£ 30000$ per QALY gained, ${ }^{11}$ restricting TKR to those patients for whom the procedure costs $<£ 20000-£ 30000 /$ QALY gained would be a reasonable approach, potentially realising savings that could be invested in other treatments giving greater health gains. However, we are aware of no published evidence assessing how the cost-effectiveness of TKR varies with OKS.

We therefore use data from the Knee Arthroplasty Trial (KAT) ${ }^{29} \quad 30$ to assess the appropriateness of rationing knee replacement by OKS, age, BMI and clinical characteristics, by calculating the cost-effectiveness of TKR in different patient subgroups. In contrast to previous studies evaluating the cost-effectiveness of TKR that relied upon data from small cohorts with short follow-up, ${ }^{6-10} 14$ the KAT trial data set provides detailed prospective individual patient data on baseline characteristics, treatments, complications, costs and quality of life and now has $>5$ years' follow-up on 2352 patients.

\section{METHODS}

We assessed the cost-effectiveness of primary TKR compared with no knee replacement for patients with different baseline characteristics. Our analysis took a NHS perspective and excluded personal and social services from the analysis, although TKR is likely to delay admission to residential care, ${ }^{14}$ reduce personal care costs and help recipients to continue paid employment. A cost-utility analysis was conducted, which assessed costeffectiveness as the cost per QALY gained to capture the quality of life gains associated with TKR.

Data on costs and quality of life following TKR were taken from the KAT trial (ISRCTN 45837371): a pragmatic, partial-factorial, unblinded randomised controlled trial in which 2352 participants attending 34 UK centres were randomly allocated to undergo TKR with/without a metal-backed tibial component, with/without patellar resurfacing and/or with/without a mobile bearing. ${ }^{29} 30$ Five-year trial results demonstrated that patients experience substantial improvements in quality of life and functional status following knee replacement that are maintained out to 5 years ${ }^{30}$; further follow-up is ongoing. Our analysis took a 5-year time horizon in line with the results published to date ${ }^{30}$; costs and health benefits occurring $>5$ years after primary TKR were conservatively excluded.

KAT participants completed the OKS and EQ-5D questionnaires immediately before and 3 months after TKR and annually thereafter. The OKS is a validated 12item questionnaire for assessing health status and outcomes in relation to knee replacement that gives an unweighted total score ranging from 0 (severe problems on all items) to 48 (no problems on any item).$^{28}$ The EQ-5D is a generic health state preference measure ${ }^{31}$; health state preference values ('utilities') for EQ-5D profiles were based on time-trade-off valuations by members of the UK general public. ${ }^{31}$ EQ-5D utilities range from -0.594 (extreme problems on all five domains) to 1 (perfect health) and indicate the value placed on different levels of health. The number of QALYs that each patient accrued following TKR was calculated as the area under the utility curve, with linear interpolation between utility measurements; those patients who died were assumed to remain at the last observed utility until death.

Healthcare resource use data (including knee-related outpatient, general practice and physiotherapy consultations and theatre time, hospital days, complications 
and knee components used during the primary admission for TKR and during any knee-related readmissions or revisions) were collected prospectively for all KAT participants and valued at 2007-2008 prices, as described previously. ${ }^{30}$ Costs incurred after year 1 were discounted at $3.5 \%$ per year based on current UK recommendations. ${ }^{32}$

The number of QALYs gained from TKR was calculated as the number of QALYs observed minus the number of QALYs expected without TKR. Since all KAT participants underwent TKR, assumptions were needed to estimate the costs and QALYs that would have been accrued if no TKR procedures had occurred in the 5-year time horizon. We assumed the following:

- Without TKR, patients would have remained at their baseline EQ-5D utility for 5 years or until death (if sooner). In practice, patients' symptoms and quality of life are likely to deteriorate due to worsening arthritis ${ }^{1733}$ and increasing age, ${ }^{34}$

- The seven KAT participants who died before hospital discharge would have survived for 5 years at baseline utility without TKR but that TKR had no effect on the date of death for other patients. This assumption is highly conservative since those patients who die soon after joint replacement tend to be older ${ }^{55}$ and have comorbidities that could have led to death without $\mathrm{TKR}^{5}$

- Patients would not have used any healthcare resources due to their knee problems if they had not undergone TKR. This assumption is highly conservative since osteoarthritis patients will receive medical management without TKR, and a recent Canadian case-control study suggested that TKR recipients accrued lower costs $6-18$ months after the procedure than matched controls who had arthritis but no TKR. ${ }^{33}$

Since these assumptions are likely to underestimate the benefits and overestimate the additional costs of TKR, we evaluated the effect of relaxing these assumptions in sensitivity analyses.

The $7 \%$ of data on baseline characteristics, resource use and quality of life that were missing were imputed by multiple imputation using the ice command ${ }^{36-38}$ (V.1.3.0) within Stata V.11.0, thereby avoiding the bias and inefficiency associated with complete case analysis. $^{38-40}$ Treatment indicators and the covariates included in subsequent regression analyses were included in the imputation model to avoid bias. ${ }^{38}$ Fifty imputed data sets were generated and results combined.

Regression models were used to estimate how the costs and QALYs gained from TKR vary with baseline characteristics and to predict outcomes for different patient groups without subdividing the trial population into small patient subgroups. Since costs and QALYs were highly skewed, generalised linear models with gamma family distributions were used to predict total costs and the QALYs accrued with or without TKR; prior to regression, the number of QALYs accrued was subtracted from the maximum number of QALYs that could have been accrued over the 5-year trial period to ensure that all QALY data were positive. We evaluated the effect of six baseline characteristics on costs and QALYs with/without TKR:

- male,

- age at time of operation,

- baseline OKS (using the new, $0-48$, scoring system ${ }^{41}$ ),

- American Society of Anaesthesiologists (ASA) grade 3 (symptomatic illness with minimal restriction on daily life) rather than grades 1 (fit and healthy) or 2 (asymptomatic illness with no restriction on daily life),

- BMI $\left(\mathrm{kg} / \mathrm{m}^{2}\right)$,

- presence of arthritis in one knee rather than in both knees or generally.

Since the introduction of biologic therapies has changed management of rheumatoid arthritis and as rheumatoid arthritis patients and those with severe comorbidities are likely have very different costs and outcomes from most patients, 108 rheumatoid arthritis patients and 13 patients with ASA grade 4 were excluded from the analysis. One hundred patients who were randomised to total versus unicompartmental knee replacement or died or withdrew from the trial before surgery were also excluded from the analysis, giving a final sample size of 2131 .

All regression analyses were conducted in Stata V.11. Bootstrapping was used to capture uncertainty around incremental cost-effectiveness ratios (ICERs) and correlations between costs and QALYs with/without TKR; the regression models for costs and outcomes were run for each of the 125 bootstrap samples drawn from each imputed KAT data set and results combined using Rubin's rule. ${ }^{39}$ Predicted costs and the predicted number of QALYs accrued with and without TKR in different patient groups were calculated from regression coefficients and used to estimate ICERs. Bootstrap results were used to calculate cost-effectiveness acceptability curves, ${ }^{42}$ which plot the probability of treatment being cost-effective against the ceiling ratio (ie, the maximum that society is willing or able to pay per QALY gained).

We also calculated the number of patients in England and Wales eligible for TKR under different rationing policies based on 61651 patients undergoing primary TKR each year. ${ }^{27}$ The proportion of patients eligible for TKR under different rationing criteria was based on analysis of an extract of patient-level Patient Reported Outcome Measures ${ }^{1}$ data on admissions for knee replacement up to 31 December 2010, supplemented where necessary by the baseline characteristics from the KAT sample.

\section{RESULTS}

Of the 2131 KAT participants who had osteoarthritis, were ASA grades $1-3$ and underwent TKR as part of the trial, the mean baseline OKS was 18 (SD: 7.5), mean 
age 71 (SD: 8.0), mean BMI 30 (SD: 5.5) and 45\% (956/2131) were men (figure 1); further baseline characteristics of the trial population have been reported previously. ${ }^{29}$

Mean EQ-5D utility rose substantially within 3 months of TKR from 0.39 at baseline to 0.71 at 1 year and declined gradually thereafter (figure 1, table 1). However, baseline utility and the quality of life gains associated with TKR varied between patient subgroups. In particular, women and patients with low OKS (ie, poor knee function), higher ASA grade or obesity at baseline tended to have lower quality of life at all timepoints (figure 1), although those in the lowest OKS quintile experienced significantly greater health gains from TKR than the top quintile $(p<0.001$; calculated using linear regression, combining imputed data sets using the $\mathrm{mim}$ command).

On average, each admission for primary TKR cost $£ 6363$ (SD: £1702). Readmissions, revision procedures and GP, outpatient and physiotherapy consultations related to the study knee over the following 5 years cost a further 11095 (SD: £3579) per patient, giving a total cost of $£ 7458$ (SD: $£ 4058$ ) per patient. TKR was more costly in patients with low baseline OKS, but the greater QALY gains meant that TKR was better value for money in this patient group than in patients with moderate symptoms (table 1). Subgroup analyses suggested that TKR cost <£11000/QALY gained across all OKS deciles: well below the $£ 20000-£ 30000 /$ QALY gained range that NICE currently consider good value for money. ${ }^{11}$
However, cost-effectiveness also varied substantially between patients within each OKS decile. We therefore used regression models to estimate the costs, QALY gains and cost-effectiveness of TKR in a wide range of patient subgroups while controlling for multiple baseline characteristics. Our results indicated that four factors have a significant effect on cost-effectiveness: age, sex, baseline OKS and ASA grade (table 2). BMI and the presence of arthritis in other joints besides the study knee had no significant effect on either the costs or QALY gains associated with TKR $(\mathrm{p}>0.05)$.

Figure 2 shows the cost-effectiveness of TKR for each multivariate group, using charts similar in design to widely used cardiovascular risk tables. ${ }^{43}$ The charts demonstrate that TKR is clearly cost-effective across a wide range of patient groups. For example, TKR costs $<£ 20000 /$ QALY gained for 71-year old women with no symptomatic illness unless baseline OKS is above 40 (equivalent to having very minor problems on up to eight of the 12 activities mentioned in OKS or moderateto-severe pain only on exertion). The threshold OKS at which TKR becomes cost-ineffective was lower for older patients, men and those with symptomatic disease, but TKR remained cost-effective for all patients with OKS below 32 regardless of age, sex or ASA grade. A simplified analysis controlling only for OKS and ASA grade suggested that TKR is cost-effective for all ASA grade 1 or 2 patients with baseline OKS $<40$, and all ASA grade 3 patients with OKS $<35$.

There was substantial uncertainty around the costeffectiveness ratios for patients with very high baseline
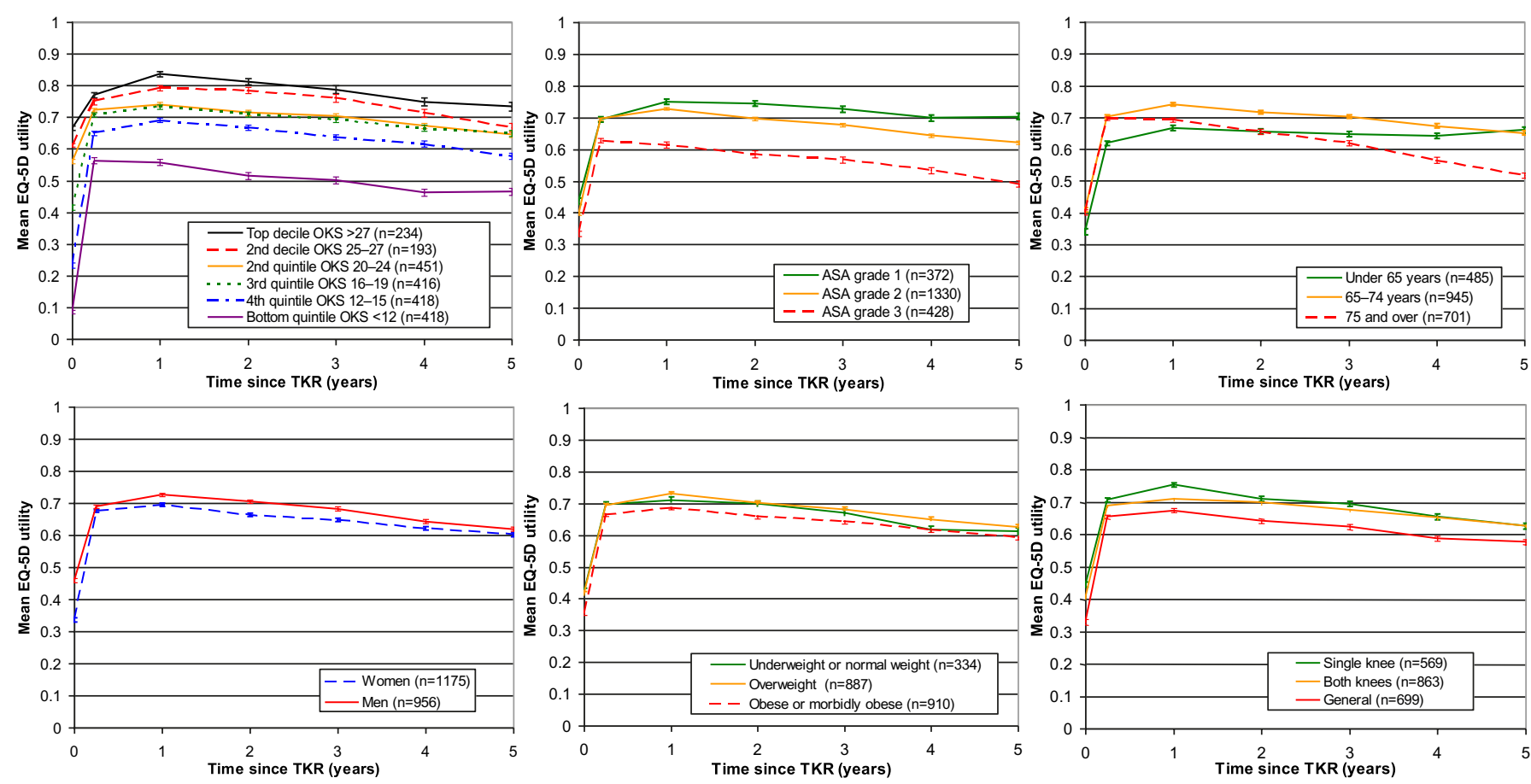

Figure 1 EQ-5D utility by subgroup. ASA, American Society of Anaesthesiologists classification (1=completely fit and healthy, $2=$ some illness but no effect on daily activity, $3=$ symptomatic illness with minimal restriction on life); OKS, Oxford Knee Score (new scoring system running from 0 (severe problems on all functions) to 48 (no problems)) ${ }^{41}$; TKR, total knee replacement. Error bars show $95 \%$ Cls. EQ-5D utility is measured on a scale from 1 (perfect health) to -0.594 , where 0 is equivalent to death. 


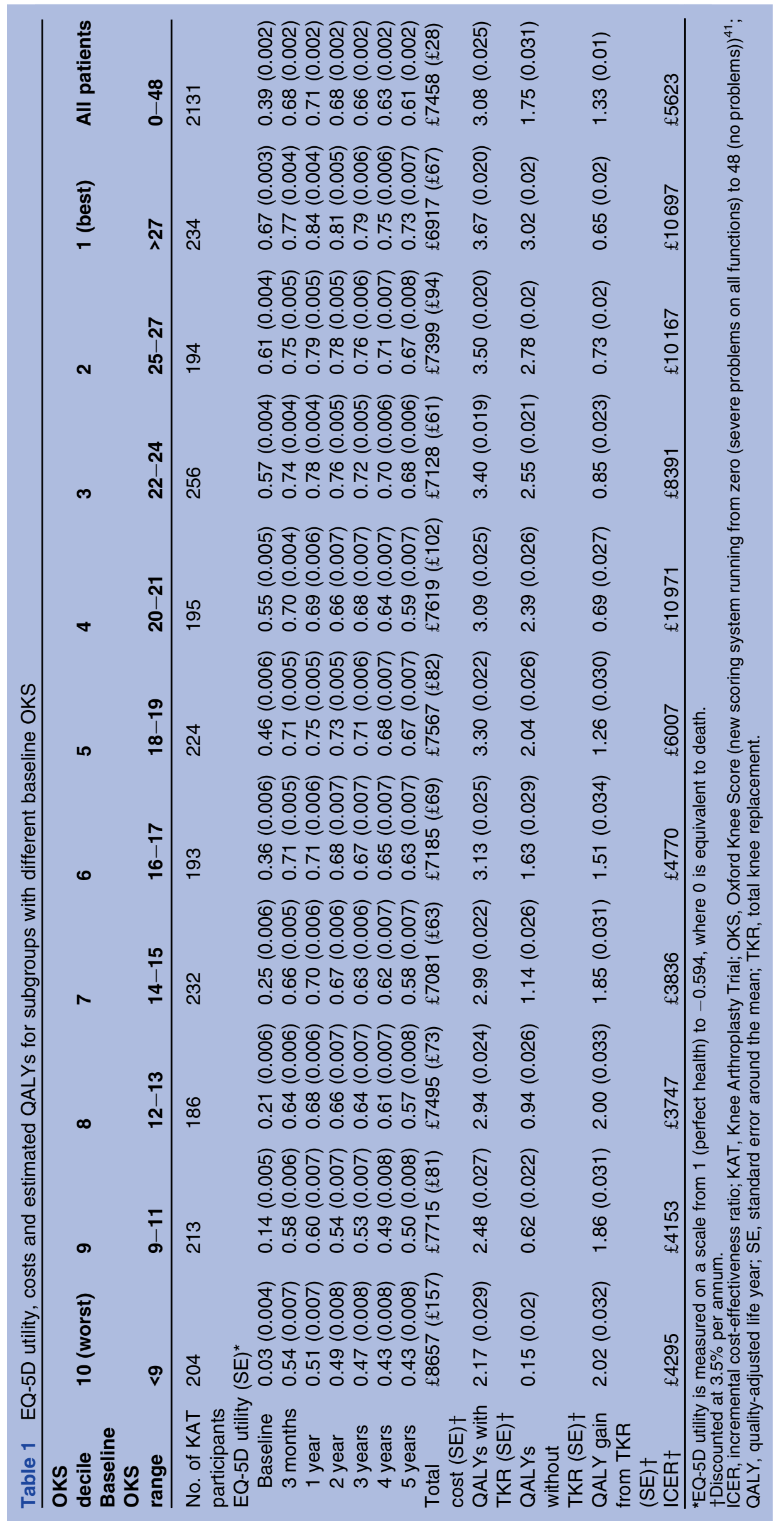


Table 2 Results of the regression analysis

\begin{tabular}{|c|c|c|c|}
\hline \multirow[b]{2}{*}{ Baseline characteristic } & \multicolumn{3}{|l|}{ Coefficient (SE) } \\
\hline & QALY loss with TKR† & QALY loss without TKR $†$ & Total costs with TKR (£) \\
\hline Male & $0.056(0.065)$ & $-0.001(0.054)$ & $593(181)^{\star}$ \\
\hline Age at operation (years) & $0.007(0.003)^{*}$ & $0.002(0.004)$ & $-8(12)$ \\
\hline Pre-operative OKS & $-0.040(0.004)^{*}$ & $-0.087(0.004)^{*}$ & $-52(12)^{*}$ \\
\hline ASA grade 3 & 0.414 & 0.2 & 492 \\
\hline Constant & $1.712(0.201)^{\star}$ & $4.274(0.325)^{\star}$ & $8573(881)^{\star}$ \\
\hline \multicolumn{4}{|c|}{ 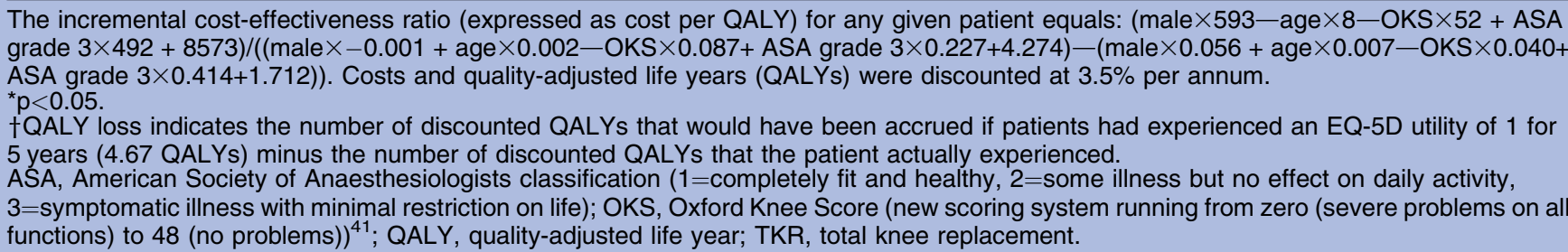 } \\
\hline
\end{tabular}

OKS since only 37 trial participants had OKS above 35 at baseline. However, we estimate that there is a $99 \%$ probability that TKR is cost-effective (at a $£ 20000 /$ QALY ceiling ratio) for the average ASA grade 1-2 patient with a baseline OKS of 35 and a $53 \%$ probability for ASA grade 3 (figure 3 ).

Since each additional baseline characteristic increases the complexity of the eligibility criteria and may also raise ethical or equity concerns, we estimated the number of patients who would receive TKR under different eligibility criteria. This analysis suggested that restricting TKR to those patients for whom the operation was predicted to cost $<£ 20000 /$ QALY gained based on the simplified model (allowing only for ASA grade and OKS) would lead to 2000 fewer TKR procedures being

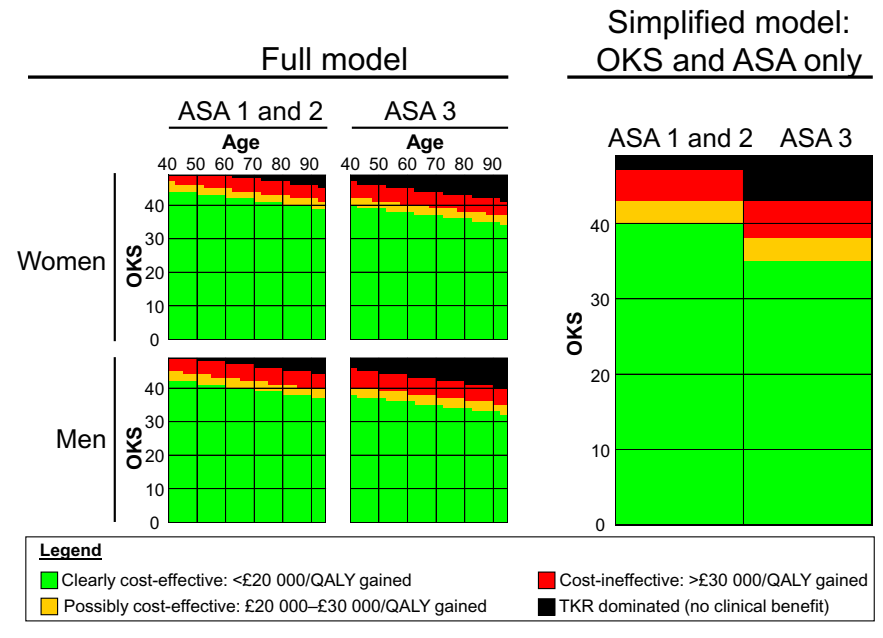

Figure 2 Cost-effectiveness prediction charts estimated based on predictions of regression models. ASA, American Society of Anaesthesiologists classification ( $1=$ completely fit and healthy, $2=$ some illness but no effect on daily activity, $3=$ symptomatic illness with minimal restriction on life); OKS, Oxford Knee Score (new scoring system running from 0 (severe problems on all functions) to 48 (no problems) ${ }^{41}$; QALY, quality-adjusted life year; TKR, total knee replacement. conducted in England and Wales each year (3\% fewer than the 61651 procedures conducted in $2009 / 2010^{27}$ ). If the decision rules were simplified further to provide TKR to all patients with baseline OKS $<39$, all but 1700 of the 61651 patients currently receiving TKR in England and Wales in $2009 / 2010^{27}$ would be eligible. Allowing for other baseline characteristics had minimal effect on patient numbers or predicted costs or benefits, suggesting that decisions about suitability for TKR can be based on OKS alone.

Sensitivity analyses were conducted to assess the effect of extending the time horizon of our analysis up to 10 years and of assuming that EQ-5D utility would have fallen by $0.5 \%$ per year without TKR, ${ }^{34}$ that all kneerelated ambulatory consultations would have occurred without TKR and that the mean length of stay has fallen by $41 \%$ (5.9 days $^{27}$ vs 10.0 ) since the KAT operations took place in the period 1999-2003. Changing any of these assumptions produced lower ICERs than the base case analysis, confirming that the current analysis is highly conservative and suggesting that TKR may be costeffective for ASA grade 1-2 patients with baseline OKS as high as 43. Excluding patients with incomplete data had no effect on conclusions. Although statistically significant, adding an age-squared term to the regression model had no effect on the conclusions, while a sensitivity analysis adding an OKS-squared term suggested that TKR may be cost-effective for all patients of ASA grade 1 or 2 . Recoding $\mathrm{BMI}$ as a dummy variable indicating whether or not patients were obese $(\mathrm{BMI} \geq 30$ ) did not change the conclusions, and obesity had no significant effect on costs or QALYs.

\section{DISCUSSION}

We found TKR to cost $£ 5623 /$ QALY gained for the average patient (similar to estimates from previous studies $^{6-10}$ ) and therefore represent very good value for money compared with the $£ 20000-£ 30000 /$ QALY threshold typically used in NHS decision making. ${ }^{11}$ Our study also confirms previous findings ${ }^{79} 17$ that costs and 

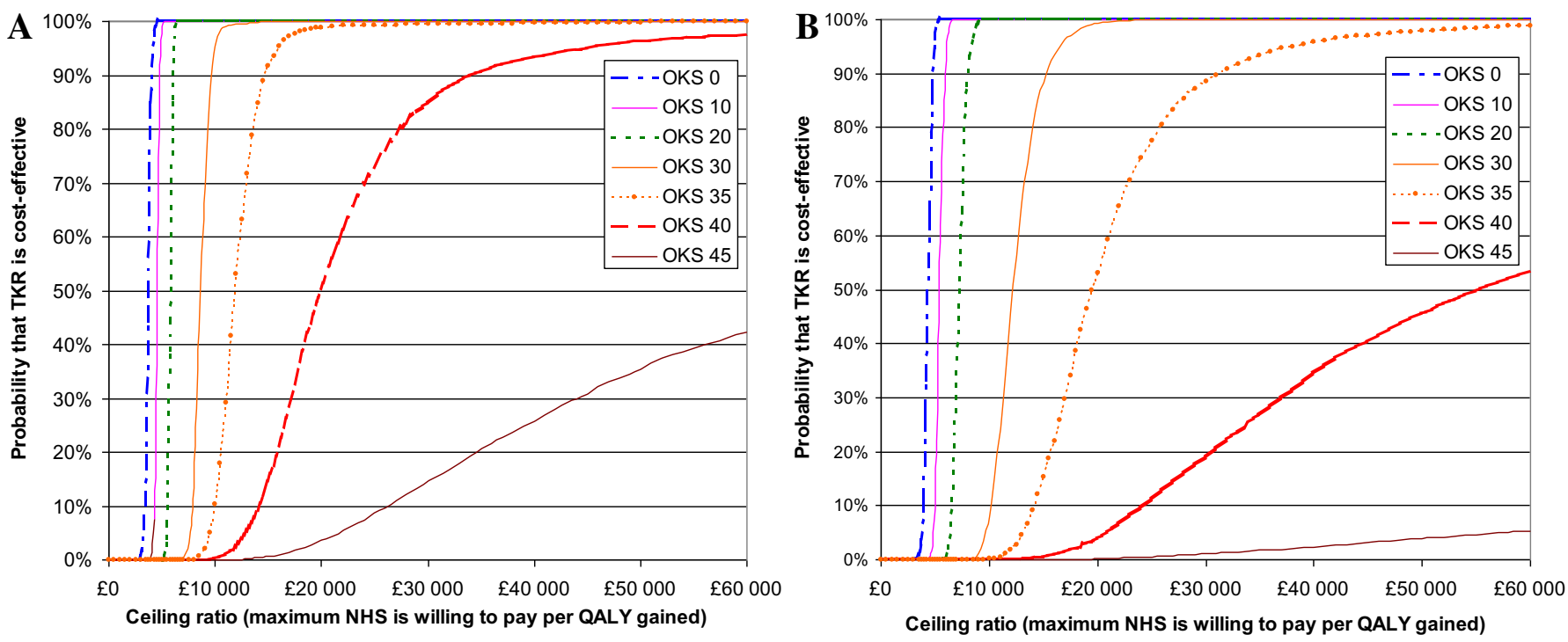

Figure 3 Cost-effectiveness acceptability curves for different patient subgroups at different baseline Oxford Knee Scores (OKS). (A) American Society of Anaesthesiologists (ASA) grade 1 and 2 patients. (B) ASA grade 3 patients.

benefits vary with age and comorbidity but evaluates such characteristics, and pre-operative knee function, in multivariate analyses, which has not (to our knowledge) been done previously. We found pre-operative OKS to be the best predictor of post-operative costs, outcomes and cost-effectiveness.

Our analyses were based on patient-level data from a large trial with detailed prospective collection of costs, utilities and baseline characteristics that permit extensive subgroup analyses. KAT used a pragmatic design with broad inclusion criteria and no restrictions on perior post-operative care other than randomised aspects of component design ${ }^{29}{ }^{30}$; as a result, the trial cohort is similar to the national Patient Reported Outcome Measures cohort, and costs and benefits are likely to be comparable to those in routine clinical practice. Furthermore, unlike some previous economic evaluations, our study took full account of uncertainty and included all knee-related NHS costs accrued in the first 5 years after TKR. The main limitation of our analysis was the small number of patients with high OKS. Since KAT recruited only 37 patients with OKS $>35$, further studies are required to evaluate TKR in this patient subgroup. Analyses were also based on post hoc comparisons between outcomes before and after TKR, rather than between randomly assigned treatment groups. As a result, our study does not provide unbiased estimates of the QALY gains from TKR, and regression towards the mean may have caused our study to overestimate the quality of life improvement attributable to TKR and/or the effect of pre-operative OKS on QALY gains.

We made several highly conservative assumptions, in particular, assuming that patients would have accrued no knee-related costs and would have remained at baseline utility without TKR, whereas previous studies have found that patients accrue substantial costs and show clinical worsening without TKR. ${ }^{33}$ We also assumed that those patients who died before hospital discharge would have survived for 5 years if they had not had TKR; in reality, those patients dying soon after TKR tend to be older ${ }^{5} 35$ and frailer ${ }^{5}$ pre-operatively, and some studies suggest that patients undergoing hip replacement have higher long-term survival than the general population ${ }^{44} 45$ (although this may be due to patient selection ${ }^{35}{ }^{44}$ ). When these assumptions were relaxed, TKR was costeffective for ASA grade 1-2 patients with baseline OKS $<44$. The costs and benefits of TKR are likely to vary between centres and over time due to variations in patient demographics, component prices, length of stay and operation time; sensitivity analyses demonstrated that TKR may have become more cost-effective since the KAT procedures were conducted in the period 1999-2003 due to reductions in length of stay.

Our analyses show that if TKR were to be rationed based on cost-effectiveness, OKS would be a reasonable tool to use to set the threshold, although there may, of course, be practical difficulties with using a patientreported measure to determine treatment eligibility: particularly if patients know how their questionnaire responses will be used or if test-retest reliability is low in this setting. The decision to operate must also take into consideration other factors, such as radiographic findings and patient choice. Based on regression and subgroup analyses, we can be confident that TKR costs $<£ 20000 /$ QALY for all ASA grade 1-2 patients with baseline OKS $<40$ and for ASA grade 3 patients with OKS $<35$. Using these thresholds to determine who received treatment would avoid around 2000 TKR procedures per year in England and Wales, saving around $£ 11.8$ million/year. However, the higher thresholds suggested by sensitivity analyses using less conservative assumptions suggest that TKR is cost-effective for all but 100 patients/year, which is unlikely to achieve 
sufficient savings to warrant rationing of TKR. Although the cost-effectiveness of knee replacement was also influenced by gender and age, taking account of these factors as well as OKS and ASA grade would increase the complexity of eligibility criteria, reduce equality of access to healthcare and have minimal effect on NHS budgets.

The results also demonstrate that the thresholds proposed by some PCTs ${ }^{18-24}$ are inappropriate and would deny a highly cost-effective treatment to thousands of patients with severe arthritis. The current situation also introduces a postcode lottery with different PCTs using different eligibility criteria. We estimate that limiting knee replacement to patients with $\mathrm{OKS} \leq 26$ would prevent $>10000$ people from receiving TKR. This would be inequitable as TKR costs $£ 10697 /$ QALY gained for this group, whereas the NICE routinely recommend treatments with ICERs in the region of $£ 20000-£ 30000 /$ QALY gained ${ }^{114647}$ and are willing to pay substantially more for end of life care. ${ }^{48}$ Furthermore, the finding that TKR and subsequent monitoring is more costly for patients with low OKS suggests that delaying TKR until symptoms have deteriorated may be a false economy. Although some PCTs have considered restricting TKR further for obese patients, ${ }^{18} 212549$ we found BMI to have no significant effect on costs $(p=0.442)$, QALY gains $(\mathrm{p}=0.098)$ or the incidence of peri- or post-operative complications $(p>0.26)$. Although BMI remained nonsignificant even when ASA grade was omitted from regression analyses, it is difficult to isolate the effect of obesity from other correlated variables (eg, comorbidity); as a result, further research on obesity may be warranted, particularly since a previous study observed a significant correlation between BMI and costs. ${ }^{13}$

Eligibility criteria for rheumatoid arthritis patients and patients with ASA grade 4 remain unclear, although ASA grade 4 patients would normally be considered unfit for surgery, and the decision to operate on such patients would be based on a case-by-case consideration of whether the benefits outweigh the risks. While our study focused on the costs and benefits of TKR, patients with high baseline OKS often undergo unicompartmental knee replacement; further research is needed to evaluate how the cost-effectiveness of unicompartmental replacement varies with $\mathrm{OKS}$.

In conclusion, TKR is highly cost-effective for the vast majority of patients who currently undergo this procedure in the UK. Although costs and health benefits vary with baseline OKS, the rationing thresholds proposed by some PCTs ${ }^{18-24}$ are not supported by evidence on health outcomes or cost-effectiveness.

Acknowledgements The authors would like to thank all those involved in the KAT trial for their role in running the trial and collecting the data used in this study, particularly other members of the KAT Project Management Group: Suzanne Breeman, Marion Campbell, Jackie Ellington, Nick Fiddian, Adrian Grant, Linda Johnston and Richard Morris. Patient Reported Outcome Measures data were reused with the permission of The Health and Social Care Information Centre, copyright 2011, with all rights reserved. The views and opinions expressed therein are those of the authors and do not necessarily reflect those of the HTA programme, NIHR, NHS, Information Centre or the Department of Health.
Funding The Knee Arthroplasty Trial is funded by the NIHR Health Technology Assessment Programme (project number 95/10/01). Additional funding for research support in clinical centres was provided by Howmedica Osteonics; Zimmer; DePuy, a Johnson and Johnson company; Corin Medical; Smith and Nephew Healthcare; Biomet Merck and Wright Cremascoli. The Health Economics Research Centre obtains support from the National Institute of Health Research. The Health Services Research Unit is core funded by the Chief Scientist Office of the Scottish Government Health Directorates. The Biomedical Research Unit at Nuffield Department of Orthopaedics, Rheumatology and Musculoskeletal Sciences is funded by the National Institute of Health Research. The authors conducted the research independently of all funding organisations. The funders had no role in the collection, analysis or interpretation of data, writing of the manuscript or the decision to publish. The authors had full access to all the data (including statistical reports and tables) in the study and take responsibility for the integrity of the data and the accuracy of the data analysis.

Competing interests All authors have completed the Unified Competing Interest form at http://www.icmje.org/coi_disclosure.pdf (available on request from the corresponding author) and declare no support from any organisation for the submitted work with the exception of those declared in the funding statement. AG, RF and GM declare that they have no financial relationships with any organisations that might have an interest in the submitted work in the previous 3 years and no other relationships or activities that could appear to have influenced the submitted work. DM receives royalties paid to him by Biomet but both declare that they have no other financial relationships with any organisations that might have an interest in the submitted work in the previous 3 years and no other relationships or activities that could appear to have influenced the submitted work.

Ethics approval The KAT trial was approved by the Multi Centre Research Ethics Committee for Scotland in November 1998 (research protocol MREC/98/0/100) and was approved by the Local Research Ethics Committees in each study centre recruiting trial participants. Further details are available on request.

Contributors HD, AG and DM conceived and developed the principles and methods underpinning the current analysis. GM was involved in primary data collection and database management, while HD conducted the analysis under AG's supervision and guidance from DM, GM and RF. HD drafted the manuscript with input from all other authors; all authors have approved the final manuscript and were involved in the interpretation of results. The KAT trial was conceived, designed and run by the KAT trial group, which comprised Suzanne Breeman, Marion Campbell, HD, Jackie Ellington, Nick Fiddian, RF, Adrian Grant, AG, Linda Johnston, GM, Richard Morris and DM.

Data sharing statement See http://bmjopen.bmj. $\mathrm{com} /$ site/about/resources/datamanagement.xhtml.

\section{REFERENCES}

1. Hospital Episode Statistics. Provisional Monthly Patient Reported Outcome Measures (PROMs) in England. April 2009 - April 2010: Pre- and Postoperative Data: Experimental Statistics. 2010. http:// www.hqip.org.uk/assets/NCAPOP-Library/PROMsreport09101.pdf (accessed 6 Apr 2011).

2. Labek G, Thaler M, Janda W, et al. Revision rates after total joint replacement: cumulative results from worldwide joint register datasets. J Bone Joint Surg Br 2011;93:293-7.

3. The National Collaborating Centre for Chronic Conditions. Osteoarthritis: National Clinical Guideline for Care and Management in Adults. London: Royal College of Physicians, 2008. http://www. nice.org.uk/nicemedia/live/11926/39720/39720.pdf (accessed 29 Jun 2011).

4. Huddleston JI, Maloney WJ, Wang Y, et al. Adverse events after total knee arthroplasty: a national Medicare study. J Arthroplasty 2009;24 (Suppl 6):95-100.

5. Memtsoudis SG, Della Valle AG, Besculides MC, et al. Risk factors for perioperative mortality after lower extremity arthroplasty: a population-based study of $6,901,324$ patient discharges. $J$ Arthroplasty 2010;25:19-26.

6. Navarro Espigares JL, Hernández Torres E. Cost-outcome analysis of joint replacement: evidence from a Spanish public hospital. Gac Sanit 2008;22:337-43.

7. Losina E, Walensky RP, Kessler CL, et al. Cost-effectiveness of total knee arthroplasty in the United States: patient risk and hospital volume. Arch Intern Med 2009;169:1113-21. discussion 1121-2. 
8. Räsänen $\mathrm{P}$, Paavolainen $\mathrm{P}$, Sintonen $\mathrm{H}$, et al. Effectiveness of hip or knee replacement surgery in terms of quality-adjusted life years and costs. Acta Orthop 2007;78:108-15.

9. Rissanen $\mathrm{P}$, Aro $\mathrm{S}$, Sintonen $\mathrm{H}$, et al. Costs and cost-effectiveness in hip and knee replacements. A prospective study. Int J Technol Assess Health Care 1997:13:575-88.

10. Lavernia CJ, Guzman JF, Gachupin-Garcia A. Cost effectiveness and quality of life in knee arthroplasty. Clin Orthop Relat Res 1997:345:134-9.

11. National Institute for Health and Clinical Excellence. Social Value Judgements: Principles for the Development of NICE Guidance. 2nd edn. 2008. http://www.nice.org.uk/media/C18/30/

SVJ2PUBLICATION2008.pdf (accessed 17 Nov 2010)

12. Brander VA, Malhotra $\mathrm{S}$, Jet $\mathrm{J}$, et al. Outcome of hip and knee arthroplasty in persons aged 80 years and older. Clin Orthop Relat Res 1997;345:67-78.

13. Dowsey MM, Liew D, Choong PF. Economic burden of obesity in primary total knee arthroplasty. Arthritis Care Res (Hoboken) 2011;63:1375-81.

14. Karuppiah SV, Banaszkiewicz PA, Ledingham WM. The mortality, morbidity and cost benefits of elective total knee arthroplasty in the nonagenarian population. Int Orthop 2008;32:339-43.

15. McQueen DA, Long MJ, Algotar AM, et al. The effect of obesity on quality-of-life improvement after total knee arthroplasty. Am J Orthop (Belle Mead NJ) 2007;36:E117-20, E127.

16. de Leeuw JM, Villar RN. Obesity and quality of life after primary total knee replacement. Knee 1998;5:119-23.

17. Cushnaghan J, Bennett J, Reading I, et al. Long-term outcome following total knee arthroplasty: a controlled longitudinal study. Ann Rheum Dis 2009;68:642-7.

18. NHS Oxfordshire. INTERIM Treatment Threshold Statement: Knee Arthroplasty (Reference PS 188). 2010. http://www.oxfordshirepct. nhs.uk/professional-resources/priority-setting/lavender-statements/ documents/PS188Kneereplacement.pdf (accessed 5 Apr 2011).

19. NHS Gloucestershire. Guidelines for Management of Orthopaedic Conditions. 2010. http://www.glospct.nhs.uk/pdf/services/ orthopaedic_referral_guidelines_\%20march\%2010v3.pdf (accessed 5 Apr 2011).

20. NHS Worcestershire. Commissioning Policy for Musculoskeletal Surgical Interventions. 2010. http://www.worcestershire.nhs.uk/ file_download.aspx?id=86bae3e2-4d88-4416-92e6-9eeda22e8208 (accessed 5 Apr 2011).

21. Milton Keynes O, Berkshire East, Berkshire West \& Buckinghamshire (MOBBB) Priorities Committee., Minutes of Meeting Held on Wednesday 15 December 2010. 2010. http://www.sph.nhs.uk/sphpsu/sph-files/mobbb-minutes/mobbb-minutes-15-december-2010 (accessed 5 Apr 2011).

22. South West London Public Health Network. 2010/11 South West London Effective Commissioning Initiative. 2010. http://www. swlphnetwork.org.uk/uploads/documents/effective-commissioningdocument-master-version-2010-11-final.doc (accessed 5 Apr 2011)

23. NHS Manchester. Topics for Inclusion or Change in the pct Noncommissioned Procedures List. 2010. http://www.manchester.nhs.uk/ document_uploads/19\%20January\%202011/C2turnaroundAppendix \%202\%20TR.doc (accessed 5 Apr 2011).

24. NHS Derbyshire County. NHS Derbyshire County Prior Approval: Procedures of Limited Clinical Value Summary. 2010. http://www.phen. nhs.uk/Derbyshire\%20County\%20Procedures\%20of\%20Limited\% 20Clinical\%20Value\%20100525\%20draft.pdf (accessed 29 Jun 2011).

25. Jeffreys B. Surgeons Raise Alarm Over Waiting. Journal [Serial on the Internet]. 2011. http://www.bbc.co.uk/news/health-12964360

26. NHS Warwickshire. Report to the PCT Board 10th November 2010 2010. http://www.warwickshire.nhs.uk/trustboardenclosures/ November2010/Enc\%208\%20In\%20Year\%20Demand\% 20Management\%20of\%20Electives\%205-11-2010.pdf (accessed 29 Jun 2011).
27. Hospital Episode Statistics. Main Procedures and Interventions: 4 Character Inpatient Data 2009-10. 2010. http://www.hesonline.nhs. uk/Ease/servlet/ContentServer?sitelD=1937\&categorylD=215 (accessed 10 Feb 2011)

28. Dawson J, Fitzpatrick R, Murray D, et al. Questionnaire on the perceptions of patients about total knee replacement. J Bone Joint Surg Br 1998;80:63-9.

29. Johnston L, MacLennan G, McCormack K, et al; KAT Trial Group. The Knee Arthroplasty Trial (KAT) design features, baseline characteristics, and two-year functional outcomes after alternative approaches to knee replacement. J Bone Joint Surg Am 2009;91:134-41.

30. Breeman $\mathrm{S}$, Campbell $\mathrm{M}$, Dakin $\mathrm{H}$, et al. Patellar resurfacing in tota knee replacement: five-year clinical and economic results of a large randomized controlled trial. J Bone Joint Surg Am 2011;93:1473-81.

31. Dolan P. Modeling valuations for EuroQol health states. Med Care 1997:35:1095-108.

32. HM Treasury. The Green Book: Appraisal and Evaluation in Central Government. London: TSO, 2003.

33. Hawker GA, Badley EM, Croxford R, et al. A population-based nested case-control study of the costs of hip and knee replacement surgery. Med Care 2009;47:732-41.

34. Kind P, Dolan P, Gudex C, et al. Variations in population health status: results from a United Kingdom national questionnaire survey. BMJ 1998;316:736-41.

35. Lie SA, Pratt N, Ryan P, et al. Duration of the increase in early postoperative mortality after elective hip and knee replacement. J Bone Joint Surg Am 2010;92:58-63.

36. Royston P. Multiple imputation of missing values. Stata Journal 2004;4:227-41

37. Royston P. Multiple imputation of missing values: further update of ice, with an emphasis on interval censoring. Stata Journal 2007:7:445-64.

38. White IR, Royston P, Wood AM. Multiple imputation using chained equations: issues and guidance for practice. Stat Med 2011;30:377-99.

39. Briggs A, Clark T, Wolstenholme J, et al. Missing... presumed at random: cost-analysis of incomplete data. Health Econ 2003:12:377-92.

40. Yu LM, Burton A, Rivero-Arias O. Evaluation of software for multiple imputation of semi-continuous data. Stat Methods Med Res 2007; $16: 243-58$.

41. Murray DW, Fitzpatrick R, Rogers K, et al. The use of the Oxford hip and knee scores. J Bone Joint Surg Br 2007;89:1010-14.

42. Fenwick E, Claxton K, Sculpher M. Representing uncertainty: the role of cost-effectiveness acceptability curves. Health Econ 2001;10:779-87.

43. British Medical Association. British National Formulary 61: Cardiovascular Risk Prediction Charts for Primary Prevention. 2011. http://bnf.org/bnf/bnf/current/204016.htm (accessed 12 Apr 2011).

44. Barrett J, Losina E, Baron JA, et al. Survival following total hip replacement. J Bone Joint Surg Am 2005;87:1965-71.

45. Lie SA, Engesaeter LB, Havelin LI, et al. Mortality after total hip replacement: 0-10-year follow-up of 39,543 patients in the Norwegian Arthroplasty Register. Acta Orthop Scand 2000;71:19-27.

46. Dakin HA, Devlin NJ, Odeyemi IA. "Yes", "No" or "Yes, but"? Multinomial modelling of NICE decision-making. Health Policy 2006;77:352-67.

47. Devlin N, Parkin D. Does NICE have a cost-effectiveness threshold and what other factors influence its decisions? A binary choice analysis. Health Econ 2004;13:437-52.

48. National Institute for Health and Clinical Excellence. Supplementary Guidance on Appraising Life-extending, End of Life Treatments. 2009. http://www.nice.org.uk/media/E4A/79/

SupplementaryAdviceTACEoL.pdf (accessed 23 Feb 2011).

49. Coombes R. Rationing of joint replacements raises fears of further cuts. BMJ 2005;331:1290. 